\title{
Evaluation of containment measures' effect on orthopaedic trauma surgery during the COVID-19 pandemic: a retrospective comparison between 2019 and 2020
}

\author{
Thibault Druel $^{1,2}$ (D) Quentin Andeol ${ }^{1,2}$ (D) Frédéric Rongieras $^{1,2,3}$ (D) Antoine Bertani $^{1,2,3} \cdot$ Maxence Bordes $^{1,2}$. \\ Agathe Alvernhe ${ }^{1,2,3}$
}

Received: 25 May 2020 / Accepted: 6 July 2020 / Published online: 21 July 2020

(C) SICOT aisbl 2020

\begin{abstract}
Background There is no available information about the effect of containment measures on trauma surgery activity. The aim of this study was to analyse and report the containment measures' impact on trauma surgery activity during the COVID-19 pandemic in order to quickly react and adjust in case of a new sanitary crisis and containment.

Methods An original epidemiological study was performed in our trauma centre in France. Data from trauma surgeries performed during the pre-containment (from March 1 to March 16, 2020), containment (from March 17 to April 17, 2020) and reference (from March 1 to April 17,2019) periods were compared. The primary outcome was the number of patients operated on daily and the daily operating room time. Clinical data, delay for surgery, mechanism of injury and injury pattern were also reviewed.

Results There was a statistically significant decrease in the number of patients operated upon daily $(-39,8 \%, p$ value $<0.001)$ and daily operating room time $(-35.5 \%, p$ value $<0.001)$ between the reference and containment periods and between the precontainment and containment periods (respectively, $-35.0 \%, p$ value $<0.001$ and $-28.7 \%, p$ value 0.002 ). No differences were reported between the reference and pre-containment periods for daily-operated patients ( $p$ value 0.359 ).

Conclusion Containment measures had a direct impact on trauma surgery activity with a decrease of a third of trauma surgery activity. Those results could be useful if a new containment occurred.
\end{abstract}

Keywords Containment measures · Pandemic · COVID-19 · Traumatology

\section{Introduction}

The COVID-19 pandemic, caused by the coronavirus SARSCoV-2 [1], is one of the largest global healthcare crises in modern history.

By May 1, 2020, France was one of the most affected country in the world: over 130,000 confirmed cases and nearly 25,000 deaths [2]. The French healthcare system has been

Level of evidence: IV, Epidemiologic

Agathe Alvernhe

agathe.alvernhe@chu-lyon.fr

1 Department of Orthopaedic and Traumatology, Edouard Herriot Hospital, 5 place d'Arsonval, 69003 Lyon, France

2 Claude Bernard University, Lyon, France

3 Department of Orthopaedic and Traumatology, Desgenettes Military Hospital, Lyon, France deeply modified to answer rapidly to the massive influx of patients.

At first glance, this medical crisis did not concern trauma surgery. The demand for specialized COVID-19 unit, an increased critical care capacity, ventilators, nurse and doctor has deprived surgical capacity. However, despite the postponement or cancellation of elective orthopaedic surgery, emergency and trauma surgery had to be performed ensuring care continuity, while adapting to the crisis context.

The pandemic also causes a severe social disruption, with the government announcement of containment measures to restrain the virus spread on March 17, 2020 [3]. The lockdown modified everyday life and activities of the French population.

Emergency activity (like type of trauma or activity volume) is a reflection of population activities. Thus, trauma surgery has been indirectly affected by the pandemic.

At the beginning, numerous data were missing to adapt our activity, owing to the unprecedented nature of the crisis. Numerous guidelines were published by national surgical 
societies [4], essentially about cancellation of elective surgery. However, we had no clue about the estimated trauma activity, and how we should organize our forces to help the COVID-19 crisis on the one hand, but still providing a quick and effective trauma activity in the operating room on the other hand.

There is not available information to predict the effect of containment measures on trauma surgery activity. In order to quickly react and adjust in case of a new containment, available data must be evaluated.

The purpose of this study was to analyse and report the containment measures' impact on trauma surgery activity in an urban academic trauma centre.

\section{Materials and methods}

\section{Study design}

This retrospective epidemiological study was conducted in the Edouard Herriot Hospital (Lyon, France) which is one of the two most important trauma centres in the county, based in an urban area of 2.3 million inhabitants. Trauma surgery for the lower and upper limbs are performed in this centre, as well as hand surgery (unique public centre in charge of emergency hand surgery in the area).

To evaluate the effect of containment measures, we compared the trauma surgery activity between 2019 and 2020. All trauma surgery data performed in our department between March 1 and April 17 in 2019 and 2020 were collected.

Three different periods were defined to investigate the effect of containment measures: the reference (from March 1 to April 17, 2019), pre-containment (from March 1 to March 16, 2020) and containment (from March 17 to April 17, 2020) periods.

The primary outcome of the study was to evaluate the containment measures' impact on our trauma activity. To this end, we calculated the daily operating room time (DORT) in hours per day and the number of patients daily operated upon. DORT is a good parameter to estimate surgical activity; it is the sum of all operating room time/24 hours. The operating room time is defined as the time between the patient's entering and leaving the operating room.

DORT was evaluated for three subgroups: specific hand trauma surgery (fractures of the wrist and below; wounds of the elbow and below) and "general" trauma surgery for the upper and lower limbs.

Medical records were also reviewed for age and sex, delay for surgery in days, mechanism of injury and injury pattern.

The mechanisms of injury were divided as follows: domestic accident (occurring at home, including sport), work-related accident, public road accident (occurring outside the home: car accident but also simple fall), suicide attempt, altercation (including domestic violence and police-related incident) and infection.
To determine the effect of containment measures on the elderly population [5], we specifically investigated proximal femur fractures.

Data of patients who underwent more than one operation (e.g. negative pressure wound therapy replacement) were only taken into account for demographics data, DORT and the number of patients daily operated and excluded for the mechanism of injury and injury pattern analysis.

\section{Statistical analysis}

Either median and quartiles or mean and standard deviation were calculated for the numeric variables. Categorical variables were reported in proportion. The Wilcoxon two-sided test was used to compare DORT and number of patients daily operated. Pearson's chi-squared test was used to compare the distribution for the mechanism of injury. Alpha risk was set to 0.05 . Statistical analysis was performed with $R \circledast$ software (version 4.0.0).

This study was conducted in accordance with the 1964 Declaration of Helsinki. This is an observational study; no ethical approval is required.

\section{Results}

A total of 520, 160 and 208 patients operated on for specific trauma injury were identified, respectively, in the reference, pre-containment and containment periods.

Background data and surgical orthopaedic trauma activity are shown in Table 1. The DORT according to specific hand trauma surgery and general trauma surgery is reported in Fig. 1.

There was a statistically significant decrease in the number of daily-operated patients $(-39.8 \%, p$ value $<0.001)$ and the DORT $(-35.5 \%, p$ value $<0.001)$ between the reference and containment periods and between the pre-containment and containment periods (respectively, $-35.0 \%, p$ value $<0.001$ and $-28.7 \%, p$ value 0.002 ). No differences were reported between the reference and pre-containment periods for the number of daily-operated patients ( $p$ value 0.359$)$ and DORT ( $p$ value 0.136 ).

The median DORT per patient was $1.6 \mathrm{~h}$ (IQR, 0.9-2.3), $1.5 \mathrm{~h}$ (IQR, 1.0-2.4) and $1.7 \mathrm{~h}$ (IQR, 1.2-2.5) with no significant difference ( $p$ value $>0.05$ in every group).

Concerning specific hand surgery, there was a statistically significant decrease only between the reference and containment periods $(-23.6 \%, p$ value 0.029$)$. There was a statistically significant decline of general trauma DORT during the containment period compared with the pre-containment ($37.6 \%, p$ value 0.001$)$ and reference $(-45.0 \%, p$ value $<$ $0.001)$ periods. No differences were found for other periods. 
Table 1 Background data and surgical orthopaedic trauma activity

\begin{tabular}{lccc}
\hline Variable & Reference period & Pre-containment period & Containment period \\
\hline Age-yearr & $47(16-98)$ & $46(16-95)$ & $49(16-99)$ \\
Mean (range, min-max) & $347(66.8)$ & $101(63.7)$ & $143(68.8)$ \\
Sex & $173(33.2)$ & $59(36.9)$ & $65(31.2)$ \\
Male - no. (\%) & $10.8( \pm 2.6)$ & $10.0( \pm 2.6)$ & $6.5( \pm 2.1)$ \\
Female - no. $(\%)$ & $18.1( \pm 4.8)$ & $12.9( \pm 4.8)$ \\
$\begin{array}{l}\text { Number of operated patients } \\
\text { Mean per day (SD) }\end{array}$ & $20.0( \pm 4.1)$ & & \\
$\begin{array}{l}\text { DORT (hours) } \\
\text { Mean per day (SD) }\end{array}$ & & & \\
\hline
\end{tabular}

SD denotes standard deviation
Figure 2 shows the evolution of trauma surgery activity in 2020 compared with 2019 , before and after the containment measures statement.

The distribution of mechanism of injury according to the different periods is reported in Fig. 3. Domestic accidents significantly increased during the containment period $(+22.3 \%$ from the reference to containment period, $p$ value $<0.05$ ). The proportion of road accidents significantly decreased from the reference to containment period ($66.7 \%, p$ value $<0.05)$. Between the containment and reference periods, there was a decrease of infection but without significant difference ( $p$ value $=0.0505$ ). There were no differences in the distribution of mechanism of injury for other periods.

The injury pattern distribution is reported in Fig. 4. The distribution of injury pattern is stable between three periods.

However, analysing proximal femur fractures in the elderly, we noticed 46 cases during the reference period (mean age 85 years, range 68-98), 12 during the pre-containment period (mean age 82 years, range 66-95) and 18 during the containment period (mean age 87 years, range 68-97). Consequently, the incidence of proximal femur fracture was 350 cases per year during the reference period, 273 cases per year during the pre-containment period ( $-22.0 \%$ compared with the reference period) and 205 cases per year during the containment period ( $-41.4 \%$ compared with the reference period and $-24.9 \%$ compared with the pre-containment period).

\section{Discussion}

\section{Surgical trauma activity}

After the containment measures began in France during the COVID-19 pandemic, our trauma surgery activity was severely impacted: we usually operate 20 hours a day, but the containment measures made our DORT drop to 12.9 hours per day. We reported a $28.7 \%$ decrease of trauma surgery activity after social activity restriction.

Note that the decrease of DORT cannot be explained by treating the most severe patients in one period. Indeed, the median DORT per patient was similar in the three periods.

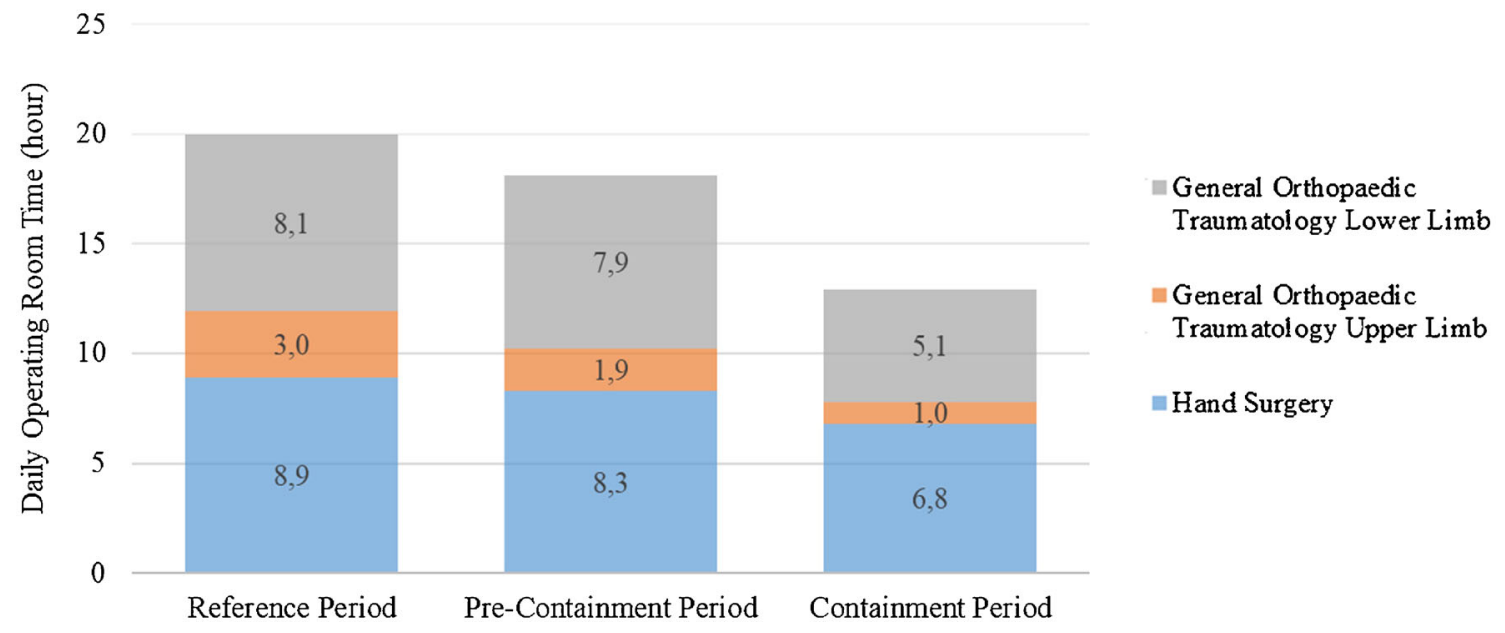

Fig. 1 Surgical orthopaedic trauma activity according to hand surgery and general orthopaedic (upper and lower limbs) 
Fig. 2 Evolution of daily operating room time before and after containment measures statement

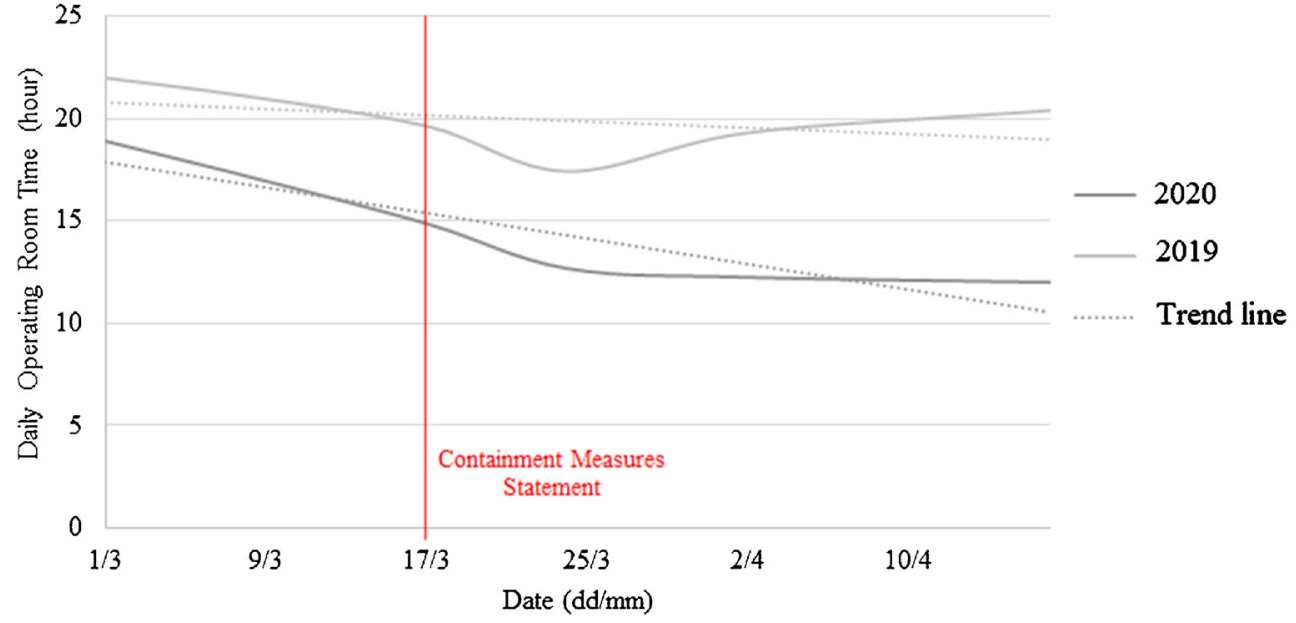

In this study, we notice that there was a trend for surgical activity decrease before the containment measures statement. The recent situation in Italy [8], the behaviour to protect themselves and others from coronavirus and the president announcement in March 12, 2020, may encourage people to be more careful before the official containment.

Surgical trauma activity in 2020 quickly decreased the first week after containment measures began, but then it seems to stabilize (Fig. 2). Thus, there is a persistent surgical trauma activity that cannot be reduced.

Considering specialized hand trauma surgery, there was an $18.1 \%$ decrease of activity after containment measures began. Considering general trauma surgery, there was a $37.8 \%$ decrease of activity after containment measures statement. Variation of trauma activity depends on people's occupation. The more important diminution of general orthopaedic trauma compared with hand trauma may be explained with the diminution of elderly traumatology and/or the diminution of road accident. Moreover, domestic accidents, which are mainly supported by hand surgeons, were higher during the containment period.

\section{Impact on the elderly}

The mortality of elderly patient with COVID-19 is higher than younger patient [9]. Because of more severe symptoms and mortality, the elderly were even more encouraged to stay at home.

The impact of the containment measures on elderly traumatology was analysed through proximal femur fractures. Indeed, proximal femur fractures are common after 65 years as reported by Singer et al. [5]. The incidence of proximal femur fractures was almost divided by two during the containment period compared with the reference period. It is the author's opinion to think that if older people went out less, all the falls at home (or in retirement homes) still occurred. As well as the global persistent surgical trauma activity discussed above, the amount of proximal femur fractures cannot be reduced to zero.
Fig. 3 Distribution of the mechanism of injury *Statistically significant differences between the containment and reference periods, $p$ value $<0.05$ $\uparrow$ Statistically significant differences between the precontainment and containment periods, $p$ value $>0.05$

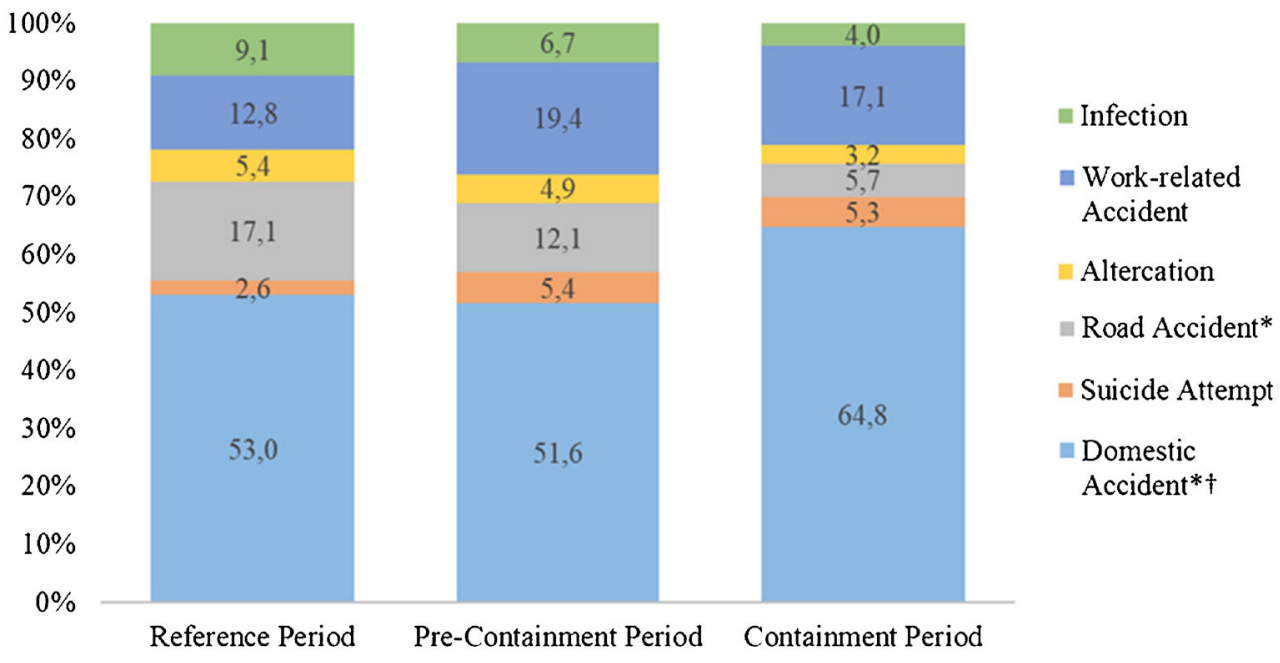




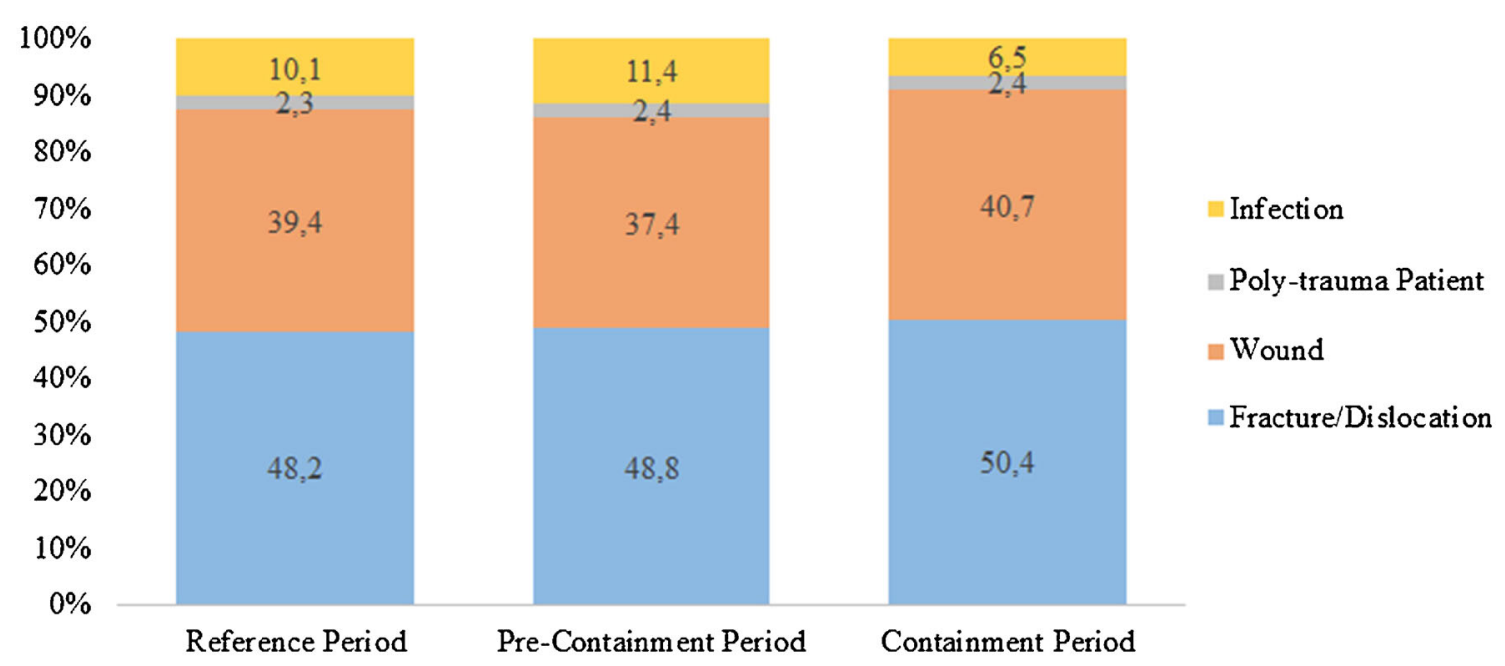

Fig. 4 Distribution of pattern injuries

\section{Mechanism of injury}

Containment measures had major consequences on social and occupational activities. Domestic accidents were by far the most common mechanism of injury, especially during the containment period. This result was expected, as well as the decrease of the road accident proportion.

Regardless of the increase of teleworking, the proportion of work-related accident was similar in the three periods. Teleworking was mostly used for office worker, whereas a large proportion of manual worker (e.g. plumber or boilermaker) continued to work. Manual workers are the most concerned by work-related accident, which explain this result.

Interestingly, there was an important decrease of the proportion of infection although no significant difference was found. The decrease of elective surgery can explain a diminution of post-operative infection but not a diminution of arthritis, cellulitis or (non-acute) prosthetic joint infection. Similar findings were found in cardiovascular department for acute coronary syndrome [10]. The probability of a causal link between the decrease of infection and containment measures is not likely. This result is concerning. It suggests that patients' infections were not diagnosed and consequently not treated. The decrease of consultation could be explained either by people's fear to go to their general practitioner or the emergency room, or by people minimizing their symptoms or even by the fear of containment controls. Another explication could be the better application of hygiene measures, or the restriction of nonsteroidal anti-inflammatory drug use [11].

\section{Forces and limitations}

The main weakness of our study is the retrospective analysis of the data. Moreover, the definition of the three groups analysed is questionable. A larger period could have been investigated but would not have been more relevant to evaluate the impact of social activity restriction. Indeed, available data is needed during this turning point to the best health system modification.

The main strength of the study is the global investigation of both general orthopaedic trauma and specialized hand surgery trauma in an important urban trauma centre. A precise description of the mechanism of injury could be reported.

During the COVID-19 pandemic, procedures were implemented in our institution. Every patient requiring surgery was tested for COVID-19 (PCR test). Only in the case of high emergency procedure (e.g. multiple trauma patients or open fractures), results of the test were not obtained before the surgery and full protections were taken. For patients who needed less urgent procedures (e.g. closed fractures or simple wounds), surgery was delayed until test results are finalized. Patients were then hospitalized in a specialized COVID-19 unit or usual unit according to test results. Results could sometime take a few hours (sometime up to $12 \mathrm{~h}$ ) due to the large number of tests performed each day in our institution. We did not compare the access time to the operating room in hours between the three periods, but it would be interesting to evaluate if all these procedures impacted care efficiency. Indeed, the goal is to minimize the spread of the virus and protect the workforce as reported by Stinner et al. [7] while continuing to be as efficient as possible to minimize the delay for surgery and the total length of hospitalization.

Finally, in addition to the containment measures' impact on trauma surgical activity, the viral activity in the country and in the area has to be considered. By May 1, 2020, the viral activity in our country was responsible for 27.8 deaths per 100,000 inhabitants and for 110.9 hospitalisations per 100,000 inhabitants [6]. If the pandemic had been even more severe in our area, there would probably have been an additional decrease in trauma activity as a result. It would be interesting to compare our results with other trauma centres in various counties according to the viral activity. 
To our knowledge, it is the first study reporting data of the evolution of surgical trauma activity due to containment measures.

\section{Conclusion}

Containment measures have a direct impact on trauma surgery activity. This study showed a $28.7 \%$ decrease of surgical trauma activity after the containment measures statement. Nevertheless, there is an amount of activity that cannot be reduced.

In the future, a third of all orthopaedic trauma resources could be reassigned to other purposes without affecting the proper functioning of the traumatology department. Every crisis is different, and these data from the COVID-19 pandemic may not be suitable to another.

\section{Acknowledgements None.}

Contribution of authors T.D.: acquisition of data, analysis and interpretation of data, writing the manuscript

Q.A.: design the study, acquisition of data, analysis and interpretation of data, writing the manuscript

F.R.: reviewing the manuscript

A.B.: reviewing the manuscript

M.B.: acquisition of data, analysis and interpretation of data, writing the manuscript

A.A.: design the study, acquisition of data, analysis and interpretation of data, writing the manuscript

Funding information This research did not receive any specific grant from funding agencies in the public, commercial or not-for-profit sectors.

\section{Compliance with ethical standards}

Conflict of interest On behalf of all authors, the corresponding author states that there is no conflict of interest.

Ethical approval This study was conducted in accordance with the 1964 Declaration of Helsinki. This is an observational study; no ethical approval is required.

\section{References}

1. Wu Y, Ho W, Huang Y et al (2020) SARS-CoV-2 is an appropriate name for the new coronavirus. Lancet 395:949-950. https://doi.org/ 10.1016/S0140-6736(20)30557-2

2. Infection au nouveau Coronavirus (SARS-CoV-2), COVID-19, France et Monde. https://www.santepubliquefrance.fr/maladies-ettraumatismes/maladies-et-infections-respiratoires/infection-acoronavirus/articles/infection-au-nouveau-coronavirus-sars-cov-2covid-19-france-et-monde. Accessed 2 May 2020

3. Décret $\mathrm{n}^{\circ} 2020-260$ du 16 mars 2020 portant réglementation des déplacements dans le cadre de la lutte contre la propagation du virus COVID-19. https://www.legifrance.gouv.fr/eli/decret/2020/3/16/ PRMX2007858D/jo/texte. Accessed 2 May 2020

4. Søreide K, Hallet J, Matthews JB et al (2020) Immediate and longterm impact of the COVID-19 pandemic on delivery of surgical services: impact of COVID-19 pandemic on delivery of surgical services. Br J Surg. https://doi.org/10.1002/bjs.11670

5. Singer BR, McLauchlan GJ, Robinson CM, Christie J (1998) Epidemiology of fractures in 15000 adults: the influence of age and gender. J Bone Joint Surg Br 80-B:243-248. https://doi.org/10. 1302/0301-620X.80B2.0800243

6. Fabacher T, Meyer N, Sauleau E Epidémie COVID-19. Groupe Méthode en Recherche Clinique CHRU Strasbourg https:// thibautfabacher.shinyapps.io/covid-france/. Accessed 2 May 2020

7. Stinner DJ, Lebrun C, Hsu JR et al (2020) The orthopaedic trauma service and COVID-19 - practice considerations to optimize outcomes and limit exposure. J Orthop Trauma. https://doi.org/10. 1097/BOT.0000000000001782

8. Placella G, Salvato D, Delmastro E et al (2020) CoViD-19 and ortho and trauma surgery: the Italian experience. Injury. https:// doi.org/10.1016/j.injury.2020.04.012

9. Liu K, Chen Y, Lin R, Han K (2020) Clinical features of COVID19 in elderly patients: a comparison with young and middle-aged patients. J Inf Secur. https://doi.org/10.1016/j.jinf.2020.03.005

10. De Filippo O, D'Ascenzo F, Angelini F et al (2020) Reduced rate of hospital admissions for ACS during Covid-19 outbreak in northern Italy. N Engl J Med. https://doi.org/10.1056/NEJMc2009166

11. Bryant AE, Bayer CR, Aldape MJ, Stevens DL (2015) The roles of injury and nonsteroidal anti-inflammatory drugs in the development and outcomes of severe group a streptococcal soft tissue infections. Curr Opin Infect Dis 28:231-239. https://doi.org/10.1097/QCO. 0000000000000160

Publisher's note Springer Nature remains neutral with regard to jurisdictional claims in published maps and institutional affiliations. 